\title{
Physics basis of Multi-Mode anomalous transport module
}

\author{
T. Rafiq, ${ }^{1}$ A. H. Kritz, ${ }^{1}$ J. Weiland, ${ }^{2}$ A. Y. Pankin, ${ }^{3}$ and L. Luo ${ }^{1}$ \\ ${ }^{1}$ Department of Physics, Lehigh University, Bethlehem, Pennsylvania 18015, USA \\ ${ }^{2}$ Departments of Applied Physics, Chalmers University of Technology and Euratom-VR Assoc., \\ S41296 Gothenburg, Sweden \\ ${ }^{3}$ Tech-X Corporation, Boulder, Colorado, USA
}

(Received 2 November 2012; accepted 13 February 2013; published online 7 March 2013)

\begin{abstract}
The derivation of Multi-Mode anomalous transport module version 8.1 (MMM8.1) is presented. The MMM8.1 module is advanced, relative to MMM7.1, by the inclusion of peeling modes, dependence of turbulence correlation length on flow shear, electromagnetic effects in the toroidal momentum diffusivity, and the option to compute poloidal momentum diffusivity. The MMM8.1 model includes a model for ion temperature gradient, trapped electron, kinetic ballooning, peeling, collisionless and collision dominated magnetohydrodynamics modes as well as model for electron temperature gradient modes, and a model for drift resistive inertial ballooning modes. In the derivation of the MMM8.1 module, effects of collisions, fast ion and impurity dilution, non-circular flux surfaces, finite beta, and Shafranov shift are included. The MMM8.1 is used to compute thermal, particle, toroidal, and poloidal angular momentum transports. The fluid approach which underlies the derivation of MMM8.1 is expected to reliably predict, on an energy transport time scale, the evolution of temperature, density, and momentum profiles in plasma discharges for a wide range of plasma conditions. (C) 2013 American Institute of Physics. [http://dx.doi.org/10.1063/1.4794288]
\end{abstract}

\section{INTRODUCTION}

A number of special modes of tokamak operation such as low confinement mode ( $L$-mode), improved $L$-mode $(I$ mode), high confinement mode ( $H$-mode), supershot, and internal transport barriers have been identified by experimentalists. The goal is to develop a theory based Multi-Mode anomalous transport module to understand the interaction between physical processes that influence transport in these different modes of tokamak operation.

The Multi-Mode module version 8.1 (MMM8.1) is an anomalous transport module consists of a combination of contributions from different transport theories. The MMM7.1 module (an earlier version of the Multi-Mode module) has been installed in the PTRANSP code, the TRANSP analysis code extended for use in carrying out predictive integrated modeling simulations, and has been used to compute thermal, particle, and toroidal angular momentum transports. The MMM7.1 is documented and organized as a stand-alone module, which fully complies with the National Transport Code Collaboration (NTCC) standards ${ }^{1}$ and is now available in the NTCC Module Library. The MMM7.1 has a single clearly defined interface, which facilitates porting the module to whole device modeling codes and in addition to PTRANSP, has been ported to Framework Application for Core-Edge Transport Simulations code (FACETS), ${ }^{2}$ Automated System for TRansport Analysis code (ASTRA), ${ }^{3}$ Tokamak Simulation Code (TSC), ${ }^{4}$ and Simulation of Radio Frequency Wave Interactions with magnetohydrodynamics (MHD) code (SWIM). ${ }^{5}$ Recently, self consistent predictive simulations are carried out using MMM7.1 module for ITER target steady state and hybrid discharges. ${ }^{6,7}$

The MMM8.1 model describes transport driven by ion temperature gradient (ITG) mode, trapped electron (TE) mode, kinetic ballooning mode (KBM), peeling mode (PM), collisionless and collision dominated MHD modes, ${ }^{8}$ electron thermal transport driven by electron temperature gradient (ETG) mode, ${ }^{9,10}$ and a model for drift resistive inertial ballooning modes (DRIBM). ${ }^{11}$ The choice of the MMM8.1 has been guided by the philosophy of using the best transport theories available for the various modes of turbulence that dominate in different regions of the plasma. The theoretical foundation of the module is improved in order to develop a better understanding of the physics of transport and more reliable extrapolations to new devices. The Weiland component of the MMM8.1 model now includes transport driven by ITG/TE/KBM/PM, collisionless and collision dominated MHD modes as well as the diffusion and radial convective pinch of toroidal and poloidal angular momentum. ${ }^{12}$ The new Weiland model has gone through a significant evolution from the model, ${ }^{13}$ used as a component in the MMM7.1 transport model, to the new Weiland component in the MMM8.1 transport model. There have been three particularly significant changes past year. (1) In addition to kinetic ballooning mode, the new Weiland model now includes the transport associated with turbulence driven by peeling modes. (2) Dependence of turbulence correlation length on flow shear is introduced in the new model to reproduce the experimental observation that anomalous transport stiffness is reduced for a combination of large flow shear and small magnetic shear. (3) The new Weiland model also includes electromagnetic effects in momentum diffusivity. The Weiland model, which is derived in shifted circular geometry, includes effects of magnetic shear, elongation, finite beta, Shafranov shift, collisions, fast ion, and impurity dilution. The poloidal width of the drift-wave eigenfunction, which can be weakly or strongly ballooning depending on the magnetic shear and other plasma parameters, is obtained 
iteratively. It has been shown that Weiland drift model can predict the observed intrinsic angular rotation of the plasma given a relatively small toroidal rotation at the edge of the plasma. Compared with older models for transport driven by Weiland drift mode model, such as the one used in the MMM95 transport model, ${ }^{14}$ the new drift mode model more accurately computes finite beta effects and the suppression of transport at low magnetic shear.

The ETG component of the MMM8.1 module includes both electrostatic and electromagnetic branches of the ETG mode $^{9,10}$ and is refined using the Jenko model threshold obtained from toroidal gyrokinetic ETG turbulence simulations. ${ }^{15}$ The ETG modes have very short wavelengths which are comparable to the electron gyro-radius and much shorter than the ion gyro-radius. Therefore, the ETG modes drive essentially only electron thermal transport, with almost no contribution to ion thermal, particle, or momentum transports.

The DRIBM component of MMM8.1 module is a generalization of resistive ballooning mode (RBM) in which two fluid drift effects and electron inertia are included. ${ }^{11}$ The derivation of the DRIBM model takes into account the parallel electron and ion dynamics, collisions, electron inertia, ion gyro-viscous stress and polarization, electron and ion temperature and density profiles, temperature and density perturbations, and diamagnetic and finite beta effects. It has been found that the DRIBM model makes essential contribution to the transport in the edge region of Ohmic and $L$-mode tokamak plasmas.

The combination of modes in MMM8.1 is necessary in order to include the variety of different physical phenomena that affect the plasma transport. These components of the MMM8.1 model provide contributions to transport in the different regions of plasma discharge. It has been found in the DIII-D, JET and TFTR $L$-mode simulations carried out using the Multi-Mode model, that the DRIBM contributes to the anomalous transport primarily near the edge of the plasma where the transport associated with ITG and TE modes are diminishing as a function of radius, while neoclassical ion thermal transport contributes mainly near the center of the discharge. The Multi-Mode model has been found to provide a better match to the temperature profiles of Ohmic and $L$-mode tokamak discharges compared to other theory-based models.

The organization of this paper is as follows. In Sec. II, the multi-fluid Weiland model equations are derived using ions, electrons and impurity continuity, and momentum and energy equations. Section III is devoted for six coupled equation for the DRIBM model, which is derived from Ohm's law, vorticity, continuity, total parallel momentum, and electron and ion energy equations. In Sec. IV, the ETG model is described. The diagonal and off-diagonal components of toroidal and poloidal momentum transport are calculated in Sec. V. The content of the paper is summarized in Sec. VI.

\section{DERIVATION OF THE WEILAND MODEL}

The fundamental equations used in the Weiland model are the reactive reduced Braginskii multi-fluid equations including charged-particle drifts due to classical collisions in the edge and turbulent collisions in the core. ${ }^{8}$ It is assumed that all of the perturbed quantities are taken to be proportional to $\exp (i \mathbf{k} \cdot \mathbf{r}-i \omega t)$, where $\mathbf{k}$ and $\omega$ are the wave vector and the frequency. The Weiland transport model is a reactive fluid model that includes the fluid resonance in the energy equation. By reactive model, it is meant that dissipation is not involved in the closure. A non-Markovian mixing length rule is used in order to separate the effects of ion modes on electron transport and vice versa. This rule is used because the Doppler shifts due to the respective magnetic drift frequencies are included in the dependencies on the real frequencies. With this choice, the transport from all instabilities on all channels can be self consistently included by adding each contribution.

\section{A. Fluid ion equations}

The continuity equation for ions with density $n_{i}$ is

$$
\frac{\partial n_{i}}{\partial t}+\nabla \cdot\left(n_{i} \mathbf{v}_{i}\right)=0
$$

where

$$
\mathbf{v}_{i}=\mathbf{v}_{E}+\mathbf{v}_{* i}+\mathbf{v}_{P i}+\mathbf{v}_{\pi i}+\hat{\mathbf{B}} v_{\| i},
$$

represents the sum of the fluid flows.

The drift velocity $\mathbf{v}_{E}$ is given by

$$
\mathbf{v}_{E}=\mathbf{E} \times \hat{\mathbf{B}} / B=\hat{\mathbf{B}} \times \nabla \phi / B=-i k_{\theta} \phi \hat{\mathbf{x}} / B,
$$

where $B=|\mathbf{B}| ; \hat{\mathbf{B}}=\mathbf{B} / B$ is the unit vector in the direction of the magnetic field; $\hat{\mathbf{x}}$ is the unit vector in the radial direction; and $k_{\theta}$ is the wave vector in the poloidal direction. The electric field vector, $\mathbf{E}$, is given in terms of the scalar potential, $\phi$, and the parallel component of vector potential, $\mathbf{A}_{\|}$, by

$$
\mathbf{E}=-\nabla \phi-\frac{\partial \mathbf{A}_{\|}}{\partial t}
$$

A low- $\beta$ approximation, that is, $\beta \equiv 2 \mu_{0} p / B^{2} \ll 1$, where $p=\left(n_{i} T_{i}+n_{e} T_{e}\right)$ is made so that the compressional magnetic field perturbation can be neglected.

The ion diamagnetic drift is given by

$$
\mathbf{v}_{* i}=\frac{\hat{\mathbf{B}} \times \nabla\left(n_{i} T_{i}\right)}{Z_{i} e n_{i} B}=\hat{\theta}\left(1+\eta_{i}\right) \omega_{* i} / k_{\theta},
$$

where $\omega_{* i}$ is the ion diamagnetic frequency, $\hat{\theta}$ is the unit vector in the poloidal direction, and $\eta_{i}$ is the ratio of ion temperature and ion density gradients. The ion diamagnetic frequency can be expressed in terms of the normalized density gradient, $g_{n i}$, that is

$$
\omega_{* i}=\frac{-k_{\theta} T_{i} g_{n i}}{Z_{i} e B R},
$$

where $g_{n i}$ is given by 


$$
g_{n i} \equiv-R \hat{\mathbf{x}} \cdot \nabla n_{i} / n_{i}
$$

where $R$ is the major radius to geometric center of the flux surface. The ratio of the ion temperature and density gradients is

$$
\eta_{i} \equiv g_{T i} / g_{n i}=\frac{n_{i} \hat{\mathbf{x}} \cdot \nabla T_{i}}{T_{i} \hat{\mathbf{x}} \cdot \nabla n_{i}}
$$

The polarization drift velocity is

$$
\mathbf{v}_{P i}=\frac{d \mathbf{E}}{d t} /\left(B \Omega_{i}\right)
$$

where

$$
\Omega_{i}=\frac{Z_{i} e B}{m_{i}}
$$

is the ion cyclotron frequency and $d / d t$ is the convective derivative.

The drift velocity due to the stress tensor $\pi_{i}$ is

$$
\mathbf{v}_{\pi i}=\frac{\hat{\mathbf{B}} \times \nabla \cdot \pi_{i}}{Z_{i} e n_{i} B},
$$

which includes the lowest order finite Larmor radius effects combined with the polarization drift resulting in the cancelation of the convective diamagnetic part of the polarization $\operatorname{drift}^{8}$ and $\mathbf{v}_{\| i}$ is the ion velocity parallel to the magnetic field. In the equations above, $e$ is the electron charge, $T_{j}$ is the temperature of species $j, m_{j}$ is the mass of species $j$, and $Z_{i}$ denotes the charge state $\left(Z_{i}=1\right.$ for hydrogen isotopes and $Z_{i}=-1$ for electrons).

Note that the $\mathbf{E} \times \mathbf{B}$ drift is electrostatic in the limit of low $\beta$. Electromagnetic effects are included in the model equations through the parallel gradient $\nabla_{\|}=\hat{\mathbf{B}} \cdot \nabla$ $=\hat{\mathbf{B}}^{(0)} \cdot \nabla+\hat{\mathbf{B}}^{(1)} \cdot \nabla$, where $\hat{\mathbf{B}}^{(0)}$ is the direction of the unperturbed magnetic field, and $\hat{\mathbf{B}}^{(1)}=\nabla \tilde{A}_{\|} \times \hat{\mathbf{e}}_{\|} / B$ is the magnetic perturbation associated with field line bending, which, in turn, is associated with the perturbed part of the parallel vector potential, $\tilde{A}_{\|}$. It follows that when the operator $\hat{\mathbf{B}}^{(1)} \cdot \nabla$ operates on any background variable $S_{0}$ (such as density, temperature, or pressure), the result has the linearized form $-i S_{0} \omega_{* S} e \tilde{A}_{\|} / T_{e}$, where $\omega_{* S}=-\left(T_{e}\right.$ $/ e B) \mathbf{k} \cdot \hat{\mathbf{B}} \times \nabla \ln S_{0}$.

Equations for divergence of the drifts are

$$
\nabla \cdot \delta\left(n_{i} \mathbf{v}_{* i}\right)=\mathbf{v}_{D i} \cdot \nabla \delta\left(n_{i} T_{i}\right) / T_{i},
$$

in which $\delta$ is used to indicate perturbed quantities

$$
\nabla \cdot \hat{\mathbf{v}}_{E}=\frac{e}{T_{i}} \mathbf{v}_{D i} \cdot \nabla \tilde{\phi}
$$

and

$$
\nabla \cdot \delta\left[n_{i}\left(\mathbf{v}_{p i}+\mathbf{v}_{\pi i}\right)\right] \approx-i n_{i} k_{y}^{2} \rho_{s i}^{2}\left[\omega-\omega_{* i}\left(1+\eta_{i}\right)\right] \frac{e \tilde{\phi}}{T_{e}},
$$

where $\tilde{\phi}$ is the perturbed electrostatic potential, $\rho_{s i} \equiv c_{s} / \Omega_{i}$ is the ion Larmor radius with the electron (rather than ion) temperature, $c_{s} \equiv \sqrt{T_{e} / m_{i}}$ is the speed of sound, and

$$
\mathbf{v}_{D i}=\frac{T_{i}}{m_{i} \Omega_{i}} \hat{\mathbf{B}} \times\left(\frac{\nabla B}{B}+\kappa\right)
$$

is the drift velocity due to $\nabla|B|$ and magnetic field curvature $\kappa=\hat{\mathbf{B}} \cdot \nabla \hat{B}$. The magnetic drift frequency $\omega_{D i}=\mathbf{k} \cdot \mathbf{v}_{D i}$ so that the linearized ion continuity equation can be written as

$$
\begin{gathered}
\left(-\omega+\omega_{D i}\right) \hat{n}_{i}+\omega_{D i} \hat{T}_{i}+\left[\left(\omega_{D i}-\omega_{* i}\right) Z_{i} T_{e} / T_{i}\right. \\
\left.-k_{\theta}^{2} \rho_{s i}^{2}\left(\omega-\omega_{* i}\left(1+\eta_{i}\right)\right)\right] \hat{\phi}+k_{\|} c_{s} \hat{v}_{\| i}=0,
\end{gathered}
$$

where the dimensionless perturbed ion density, ion temperature, electrostatic potential, and parallel ion flow velocity, $\hat{n}_{i} \equiv \tilde{n}_{i} / n_{i}, \hat{T}_{i} \equiv \tilde{T}_{i} / T_{i}, \hat{\phi} \equiv e \tilde{\phi} / T_{e}$, and $\hat{v}_{\| i} \equiv \tilde{v}_{\|_{i}} / c_{s}$ are defined in terms of the perturbed variables, $\tilde{n}_{i}, \tilde{T}_{i}, \tilde{\phi}$, and $\tilde{v}_{\| i}$.

The parallel ion motion $v_{\| i}$ is determined by the parallel ion momentum equation driven by electromagnetic forces as well as by the ion pressure gradient and momentum transfer along the field lines with the ion stress tensor and zero order background flow included

$$
m_{i} n_{i}\left(\partial_{t}+2 \mathbf{v}_{D i} \cdot \nabla\right) \delta v_{\| i}=-m_{i} n_{i} \delta \mathbf{v}_{E} \cdot \nabla V_{\| 0}-\left[\hat{\mathbf{e}}_{\|} \cdot \nabla+V_{\| 0} \frac{m_{i} \mathbf{v}_{D i} \cdot \nabla}{T_{i}}\right] \times\left(\delta p_{i}+e n_{i} \delta \phi-\frac{\omega-\omega_{* i}\left(1+\eta_{i}\right)}{c k_{\|}} e n_{i} \delta A_{\|}\right)
$$

After Fourier transformation, Eq. (17) takes the form

$$
\begin{aligned}
\left(\omega-2 \omega_{D i}\right) \hat{v}_{\| i}= & -k_{\theta} \rho_{s} \frac{d V_{\| 0}}{d r} \hat{\phi}+\left[Z_{i} k_{\|} c_{s}+\frac{T_{e}}{T_{i}} \hat{V}_{\| 0} \omega_{D i}\right] \\
& \times\left[\hat{\phi}+\frac{T_{i}}{T_{e}}\left(\hat{n}_{i}+\hat{T}_{i}\right)-\frac{\omega-\omega_{* i}\left(1+\eta_{i}\right)}{c k_{\|}} \hat{A}_{\|}\right],
\end{aligned}
$$

where $\hat{A}_{\|} \equiv e \tilde{A}_{\|} / T_{e}$ is the dimensionless form of the parallel component of the perturbed vector potential, $\tilde{A}_{\|}$and $\hat{V}_{\| 0}$ is the equilibrium flow normalized by the sound velocity.
The ion energy balance equation is

$$
\begin{aligned}
& \frac{3}{2} n_{i}\left(\partial / \partial t+\mathbf{v}_{i} \cdot \nabla\right) T_{i}+n_{i} T_{i} \nabla \cdot \mathbf{v}_{i} \\
& =-\nabla \cdot \mathbf{q}_{* i}=\frac{5}{2} n_{i}\left(\mathbf{v}_{* i}-\mathbf{v}_{D i}\right) \cdot \nabla T_{i}
\end{aligned}
$$

where

$$
q_{* i}=\frac{5 n_{i} T_{i}}{2 m_{i} \Omega_{i}}\left(\hat{\mathbf{B}} \times \nabla T_{i}\right)
$$


is the diamagnetic ion heat flow. When continuity equation is utilized, the diamagnetic part of $\nabla \cdot q_{* i}$ is canceled by the convective diamagnetic terms from $v_{* i} \cdot \nabla T$ and $\nabla \cdot v_{* i}$, and Eq. (19) can be expressed in the following form: ${ }^{8}$

$$
\left(-\omega+\frac{5}{3} \omega_{D i}\right) \hat{T}_{i}+\frac{2}{3} \omega \hat{n}_{i}+\omega_{D e} \frac{g_{n i}}{2}\left(\eta_{i}-\frac{2}{3}\right) \hat{\phi}=0 .
$$

\section{B. Electron equations}

The electrons can be divided into two classes: Trapped (with density $n_{e t}$ and fraction $f_{t}=n_{e t} / n_{e}$ ) and free (with density $n_{e f}$ and fraction $1-f_{t}$ ) with $n_{e}=n_{e t}+n_{e f}$. The electron density $n_{e}$ is related to the density of hydrogenic ions $n_{H}$, impurity ions $n_{Z}=f_{Z} n_{e}$, and superthermal hydrogenic ions $n_{s}=f_{s} n_{e}$ through charge neutrality $n_{e}=n_{H}+Z_{Z} n_{z}+n_{s}$, where $Z_{Z}$ is the charge state for the impurity ions. The normalized perturbed densities (such as $\hat{n}_{e}=\tilde{n}_{e} / n_{e}$ ) are then related by

$$
\hat{n}_{e}=f_{t} \hat{n}_{e t}+\left(1-f_{t}\right) \hat{n}_{e f}=\left(1-Z_{Z} f_{Z}-f_{s}\right) \hat{n}_{H}+Z_{Z} f_{Z} \hat{n}_{Z},
$$

assuming that superthermal ions do not take part in the perturbation, i.e., $\hat{n}_{s}=0$.

The continuity equation for trapped electrons is derived from a kinetic equation including a Bhatnagar-Gross-Krook (BGK) collision term for trapped particles, in the limit $\omega \ll \Omega_{e}$, and ignoring electron finite Larmor radius effects ${ }^{16}$

$$
\frac{\partial n_{e t}}{\partial t}+\nabla \cdot n_{e t}\left(\mathbf{v}_{E}+\mathbf{v}_{* e}\right)=-\hat{\nu} \omega_{D e}\left(n_{e t}-\Gamma n_{e t} \frac{e \phi}{T_{e}}\right),
$$

where

$$
\Gamma=1+\frac{g_{T e}}{\omega / \omega_{D e}+i \hat{\nu}-1} \quad \text { and } \quad \hat{\nu} \equiv\left(\nu_{e} / \omega_{D e}\right) R / r .
$$

The electron collision frequency $\nu_{e}$ is given by

$$
\nu_{e}=4(2 \pi)^{1 / 2} n_{e}(\ln \lambda) e^{4} Z_{e f f} /\left[3\left(4 \pi \epsilon_{o}\right)^{2} m_{e}^{1 / 2}\left(k_{b} T_{e}\right)^{3 / 2}\right],
$$

where $\lambda$ is the Coulomb logarithm, $Z_{\text {eff }}$ is the effective charge, $\epsilon_{o}$ is the permittivity of free space, and $k_{b}$ is the Boltzmann constant.

The electron diamagnetic velocity $\mathbf{v}_{* e}$ (including the full electron pressure gradient) is

$$
\mathbf{v}_{* e}=-\frac{\hat{\mathbf{B}} \times \nabla\left(n_{e} T_{e}\right)}{e n_{e} B}=\hat{\theta}\left(1+\eta_{e}\right) \omega_{* e} / k_{\theta},
$$

where

$$
\omega_{* e}=\frac{k_{\theta} T_{e} g_{n e}}{e B R} \quad \text { and } \quad \eta_{e} \equiv g_{T e} / g_{n e}=\frac{n_{e} \hat{\mathbf{x}} \cdot \nabla T_{e}}{T_{e} \hat{\mathbf{x}} \cdot \nabla n_{e}} .
$$

Equations for divergence of the drifts are

$$
\begin{aligned}
& \nabla \cdot \delta\left(n_{e t} \mathbf{v}_{* e}\right)=i n_{e t} \omega_{D e}\left(\hat{n}_{e t}+\hat{T}_{e t}\right), \\
& \nabla \cdot \delta\left(n_{e t} \mathbf{v}_{E}\right)=i n_{e t}\left(\omega_{* e}-\omega_{D e}\right) \hat{\phi},
\end{aligned}
$$

in which $\omega_{D e}=\mathbf{k} \cdot \mathbf{v}_{D e}$ is electron magnetic drift frequency, $\hat{n}_{e t} \equiv \tilde{n}_{e t} / n_{e t}, \hat{T}_{e t} \equiv \tilde{T}_{e t} / T_{e t}$. With the use of the divergence expressions in Eqs. (28) and (29), the linearized continuity equation for trapped electrons, Eq. (23), can be written as follows:

$$
\left(\omega-\omega_{D e}+i \hat{\nu} \omega_{D e}\right) \hat{n}_{e t}+\left(\omega_{D e}-\omega_{* e}\right) \hat{\phi}=\omega_{D e} \hat{T}_{e t}+i \hat{\nu} \omega_{D e} \Gamma \hat{\phi}
$$

The trapped electron temperature is determined by the following electron energy equation:

$$
\begin{aligned}
& \frac{3}{2}\left(\frac{\partial}{\partial t}+v_{e t} \cdot \nabla\right)\left(n_{e t} T_{e t}\right)+\frac{5}{2} n_{e t} T_{e t} \nabla \cdot \mathbf{v}_{e t}+\nabla \cdot \mathbf{q}_{e} \\
& \quad=-\zeta \nu_{t h} T_{e t}\left(n_{e t}-n_{e t} \frac{e \phi}{T_{e}}\right),
\end{aligned}
$$

where $\nu_{t h}=\nu_{e} / \epsilon, \epsilon$ is the inverse aspect ratio and where a free parameter $\zeta$ is introduced in the energy equation in order to compensate the fact that velocity dependence on collision frequency is changed. With the cross-field (or Righi-Leduce) electron heat flux

$$
\mathbf{q}_{e}=-\frac{5}{2} \frac{n_{e t} T_{e t}}{e B} \hat{\mathbf{B}} \times \nabla T_{e t} .
$$

Equation (31) for the trapped electron temperature, through use of trapped electron continuity equation, Eq. (23), and by canceling the convective diamagnetic effects, reduces to

$$
\begin{aligned}
& \frac{3}{2} n_{e t}\left(\frac{\partial}{\partial t}+v_{E} \cdot \nabla\right) T_{e t}-T_{e t}\left(\frac{\partial}{\partial t}+v_{E} \cdot \nabla\right) n_{e t}+\frac{5}{2} n_{e t} v_{D e} \cdot \nabla T_{e t} \\
& \quad=\nu_{t h} T_{e t} n_{e t}\left(\left(\zeta-\frac{5}{2} \Gamma\right) \hat{\phi}-\left(\zeta-\frac{5}{2}\right) \hat{n}_{e t}\right)
\end{aligned}
$$

which further simplifies to the following form:

$$
\begin{aligned}
\left(\omega-\frac{5}{3} \omega_{D e}\right) \hat{T}_{e t}= & {\left[\omega_{* e}\left(\eta_{e}-\frac{2}{3}\right)+i \nu_{t h}\left(\frac{2}{3} \zeta-\frac{5}{3} \Gamma\right)\right] \hat{\phi} } \\
& +\frac{2}{3}\left(\omega+i \nu_{t h}\right) \hat{n}_{e t} .
\end{aligned}
$$

A comparison with typical terms by Braghinskii indicates that $\zeta=1.5 .^{16}$

The free electron continuity equation is

$$
\frac{\partial n_{e f}}{\partial t}+\nabla \cdot n_{e f}\left(\mathbf{v}_{E}+\mathbf{v}_{* e}+v_{\| e 0} \frac{\delta \mathbf{B}_{\perp}}{B}+\mathbf{v}_{\| e}\right)=0,
$$

which can be rewritten as

$$
\begin{aligned}
\frac{\partial \tilde{n}_{e f}}{\partial t} & +n_{0}\left(\mathbf{v}_{* e}-\mathbf{v}_{D e}\right) \cdot \nabla \hat{\phi}+\mathbf{v}_{D e} \cdot \nabla \tilde{n}_{e f}+n_{e f} \mathbf{v}_{D e} \cdot \nabla \hat{T}_{e} \\
& -\frac{1}{e} \frac{\tilde{\mathbf{B}}_{\perp}}{B} \cdot \nabla J_{\| 0}+n_{0} \hat{\mathbf{e}}_{\|} \cdot \nabla \tilde{v}_{\|}=0 .
\end{aligned}
$$

With the use of the following equation:

$$
\frac{\tilde{\mathbf{B}}_{\perp}}{B} \cdot \nabla J_{\| 0}=\frac{\partial J_{\| 0}}{d r} \frac{1}{B r} \frac{\partial \tilde{A}_{\|}}{\partial \theta}
$$


and with the assumption of a plane wave, the equation for the free electron density, Eq. (36), can be rewritten as

$$
\begin{aligned}
\left(\omega-\omega_{D e}\right) \hat{n}_{e f}= & \left(\omega_{* e}-\omega_{D e}\right) \hat{\phi}-\frac{k_{\theta}}{e n_{0} B r} \frac{\partial J_{\| 0}}{\partial r} \tilde{A}_{\|} \\
& +\omega_{D e} \hat{T}_{e f}+k_{\|} \tilde{v}_{\| e},
\end{aligned}
$$

where $\hat{n}_{e f} \equiv \tilde{n}_{e f} / n_{e f}$ and $\hat{T}_{e f} \equiv \tilde{T}_{e f} / T_{e f}$. A relation between the perturbed free (circulating) electron density $n_{e f}$ and the perturbed electric and magnetic potentials can be obtained from the momentum equation for free electrons parallel to the unperturbed magnetic field

$$
\begin{aligned}
m_{e} \frac{\partial v_{\| e}}{\partial t}= & e\left(\hat{\mathbf{B}} \cdot \nabla \phi+\frac{1}{c} \frac{\partial A_{\|}}{\partial t}\right)-\frac{e}{c}\left(\mathbf{v}_{* e} \times \delta \mathbf{B}_{\perp}\right) \cdot \hat{\mathbf{B}} \\
& +\frac{1}{n_{e f}}\left(R_{e i}-\hat{\mathbf{e}}_{\|} \cdot \nabla p\right),
\end{aligned}
$$

where $R_{e i}=j_{\|} m_{e} \nu_{e f} / 1.96 e$ is the momentum gained by electrons through collisions with ions in which $j_{\| e}=-e n_{e f} \tilde{v}_{\| e}$. With the assumptions that electron velocity parallel to magnetic field is much greater than the parallel ion velocity and that $\nu_{e} \gg \omega$, the perturbed electron velocity gives

$$
\hat{n}_{e f}=\hat{\phi}-\hat{T}_{e f}+\frac{\left(1+\eta_{e}\right) \omega_{* e}-\omega}{c k_{\|}} \hat{A}_{\|}-\frac{\tilde{v}_{\| e}}{i k_{\|} D_{e}},
$$

where $D_{e} \equiv 2 T_{e} / m_{e} \nu_{e}$.

The free electron temperature is assumed to be isothermal so that

$$
\hat{T}_{e f}=\eta_{e} \frac{\omega_{* e}}{c k_{\|}} \hat{A}_{\|} .
$$

With the use of the toroidal component of Ampere's law

$$
J_{\| 0}=\frac{1}{\mu_{0}} \frac{1}{r} \frac{\partial}{\partial r}\left(r B_{\theta}\right)
$$

and Eqs. (38), (40), and (41), the following relation between normalized electrostatic potential $(\hat{\phi})$ and vector potential $\left(\hat{A}_{\|}\right)$is obtained:

$$
\begin{aligned}
\left(\omega-\omega_{* e}\right) \hat{\phi}= & \frac{1}{c k_{\|}}\left[\omega\left(\omega-\omega_{* e}\right)+\omega_{D e}\left(\omega_{* e T}-\omega\right)\right. \\
& -k_{\|} k_{\theta} \rho_{s}^{2} v_{A}^{2} \frac{1}{B}\left(\frac{1}{r} \frac{\partial B_{\theta}}{\partial r}-\frac{B_{\theta}}{r^{2}}+\frac{\partial^{2} B_{\theta}}{\partial r^{2}}\right) \\
& \left.-k_{\perp}^{2} \rho_{s}^{2} k_{\|}^{2} v_{A}^{2}-i \omega_{D e}^{2} \frac{m_{e}}{m_{i}} \frac{R}{c_{s}} \nu_{e} \frac{k_{\perp}^{2}}{\beta_{e}} \rho_{s}\left(1-\frac{\omega}{\omega_{D e}}\right)\right] \hat{A}_{\|},
\end{aligned}
$$

where $\omega_{* e T}$ is the diamagnetic drift frequency with temperature gradient, $v_{A}^{2} \equiv B^{2} / \mu_{0} n m_{i}$ is the Alfvén speed, and $\beta_{e} \equiv$ $\mu_{0} n T_{e} / B^{2}$ is the ratio of electron pressure to magnetic pressure.

The third term in bracket on the RHS of Eq. (43) contains the poloidal magnetic field $\left(B_{\theta}\right)$ and its gradient. This term results in the inclusion of the peeling mode and is a consequence of the background current gradient which appears in Eq. (38). The inclusion of the peeling mode in the
Weiland model is important for the simulation of the edge transport barrier (ETB). The dependence of ETB on $B_{\theta}$ has been seen in previous studies. ${ }^{8}$ The height of the ETB has been found to increase with an increase in $B_{\theta}$. This is because $B_{\theta}$ is linked with background current gradient and an increase in the value of $B_{\theta}$ activates peeling mode effects in the Weiland model. ${ }^{8}$ The contribution of the peeling modes will be important in instances where there is a strong current gradient. Consequently, it is anticipated that the peeling modes will contribute in the $\mathrm{H}$-mode pedestal region.

The fifth (last) term in the bracket on the RHS of Eq. (43) includes the effects of the collisions on free electrons. In the earlier version of the Multi-Mode model, ${ }^{14}$ the equation comparable to Eq. (43) did not include the third and fifth terms in the bracket. The presence of collisions on free electrons in the Weiland model does include resistive ballooning modes. Therefore, a detailed validation study will be carried out in the future once the MMM8.1 module is installed in a predictive integrated modeling code. The result of that validation study will be to eliminate the double counting of the effects of the contribution from the Weiland component and from the DRIBM component.

\section{Impurity equations}

The impurity equations are analogous to the ion equations, continuity Eq. (16), momentum Eq. (18), and energy Eq. (21). The impurity particle equation is

$$
\begin{gathered}
\left(-\omega+\omega_{D Z}\right) \hat{n}_{Z}+\omega_{D Z} \hat{T}_{Z}+\left[\left(\omega_{D Z}-\omega_{* Z}\right) Z T_{e} / T_{Z}\right. \\
\left.-k_{\theta}^{2} \rho_{s Z}^{2}\left(\omega-\omega_{* Z}\left(1+\eta_{Z}\right)\right)\right] \hat{\phi}+k_{\|} c_{s z} \hat{v}_{\| Z}=0 .
\end{gathered}
$$

The impurity momentum equation parallel to the magnetic field is

$$
\begin{aligned}
\omega \hat{v}_{\| Z}= & Z k_{\|} c_{s z}\left[\hat{\phi}+\left(\frac{\omega_{* Z}\left(1+\eta_{Z}\right)-\omega}{c k_{\|}}\right) \hat{A}_{\|}\right] \\
& +\frac{T_{Z}}{T_{e}} c_{s z} k_{\|}\left(\hat{n}_{Z}+\hat{T}_{Z}\right) .
\end{aligned}
$$

The impurity energy equation is

$$
\left(-\omega+\frac{5}{3} \omega_{D Z}\right) \hat{T}_{Z}+\frac{2}{3} \omega \hat{n}_{Z}+\omega_{D e} \frac{g_{n Z}}{2}\left(\eta_{Z}-\frac{2}{3}\right) \hat{\phi}=0 .
$$

\section{Evolution of the Weiland model}

The new Weiland model for drift modes has gone through a significant evolution from the model and has been used as a component in the first release of Multi-Mode transport model, ${ }^{14}$ to the new Weiland component in the MMM8.1 transport model. There have been four particularly significant changes: First, eigenfunctions have been allowed to extend along magnetic field lines so that the geometrical shapes of the magnetic surfaces play a more significant role in the model. Second, toroidal and poloidal momentum transports are computed (see Sec. IV below). Third, collisions on free electrons and plasma current gradients are 
added to describe the edge physics in the new Weiland model. Fourth, the dependence of flow shear on the correlation length (length-scale of turbulence) is introduced in the new model to reproduce the experimental observation that stiffness is reduced for a combination of large flow shear and small magnetic shear.

An effect of the extended eigenfunctions is illustrated by the following partial derivation. Note that the magnitude of the ion magnetic drift frequency varies strongly around each magnetic surface

$$
\omega_{D j}=\frac{-2 k_{\theta} T_{j}}{Z_{j} e B R} g(\theta),
$$

where for Shafranov-shifted circular magnetic surfaces,

$$
g(\theta)=\cos \theta+s \theta \sin \theta+\alpha_{m} \sin ^{2} \theta .
$$

In Eq. (48), $s$ is the magnetic shear and $\alpha_{m}$, given by

$$
\alpha_{m}=\frac{2 \mu_{0} R q^{2}}{B^{2}} \frac{d p}{d r}
$$

is proportional to the Shafranov shift of the magnetic surfaces relative to one another. Consider eigenfunctions that are extended along each magnetic field line with a poloidal angular dependence that is proportional to $\exp \left(-\alpha \theta^{2}\right)$. The coefficient $\alpha$ is determined by asymptotically matching the eigenfunction solution at large poloidal angle, $\theta \rightarrow \infty$, and is given by

$$
\alpha=|\operatorname{Re}(\omega)| k_{\theta}^{2} \rho_{s}^{2}|s| q / \omega_{D e}
$$

where $\omega$ is the eigenvalue (frequency and growth rate) associated with the mode. The flux-surface average of the geometric factor, $g(\theta)$ defined in Eq. (48), times the eigenfunction, $\exp \left(-\alpha \theta^{2}\right)$, has the form

$$
\begin{aligned}
\left\langle g(\theta) \exp \left(-\alpha \theta^{2}\right)\right\rangle= & \left(1+\frac{s}{4 \operatorname{Re}(\alpha)}\right) \exp \left(-\frac{1}{8 \operatorname{Re}(\alpha)}\right) \\
& +\frac{\alpha_{m}}{2}\left[1-\exp \left(-\frac{1}{8 \operatorname{Re}(\alpha)}\right)\right]
\end{aligned}
$$

The coefficient $\alpha$ and, therefore, the eigenvalue $\omega$, appear in the denominator and exponential function in this flux-surface average. Since this kind of flux-surface average appears in several of the eigenvalue equations in the Weiland model, it can be seen that the eigenvalue equations are no longer linear in the new Weiland model. It is necessary to solve nonlinear equations for the frequencies and growth rates of the eigenfunctions. The solution is determined by carrying out a nonlinear iteration of the eigenvalue equations. It is found that the eigenfunctions extend particularly far along magnetic field lines in regions of low magnetic shear in the core of the plasma (weak ballooning approximation) while the eigenfunctions are concentrated near the midplane $(\theta=0)$ in regions of high magnetic shear which occur near the edge of the plasma (strong ballooning approximation).
Inclusion of eigenfunctions that can extend along the field line is a feature that was not implemented in the previously published description of the Multi-Mode transport model $^{14}$ but is now a new feature in the MMM8.1 transport model. Usually for small positive or for reverse magnetic shear, eigenfunctions of drift modes become extended along the field line and growth rates of these modes decrease and as a consequence the temperature increases. Therefore, inclusion of the extended eigenfunction in the Multi-Mode module is important in order to simulate discharges with internal transport barriers in the temperature profiles.

Scalings of the correlation length of drift wave turbulence with magnetic $q$, shear, elongation, temperature ratio, and flow shear have been introduced into the new Weiland drift wave transport model. The overall numerical scalings have resulted in the following numerical fit for the correlation length: ${ }^{17,18}$

$$
k_{r} \rho_{s}=\sqrt{\frac{2 K}{1+T_{i} / T_{e}}},
$$

where

$$
\begin{array}{ll}
K=\left(0.7+C\left|\hat{\omega}_{e \times b}\right|(1-|\hat{s}|)^{2}+\frac{2.4}{7.14 q \hat{s}+0.1}\right)\left(k_{\theta} \rho_{s}\right)^{2}, \\
C=4+3(\hat{s}-0.2) / 0.2 & 0 \leq \hat{s}<1, \\
C=0 & \hat{s} \geq 1 .
\end{array}
$$

The $\hat{\omega}_{e \times b}$ is the flow shear normalized by magnetic drift frequency and $\hat{s}=\sqrt{2 s-1+\kappa^{2}(s-1)^{2}}$ indicates the modification of magnetic shear $s$ due to elongation $\kappa$. The value of the Larmour radius parameter, $k_{\theta} \rho_{s}$ is typically taken as 0.33 . The plasma elongation mostly modifies the behavior of the MHD modes, so that their beta threshold increases with increasing elongation. The effects of elongation have been studied in gyrokinetic ITG/TEM turbulence simulations in Ref. 19. The effect of $\mathbf{E} \times \mathbf{B}$ flow shear, which reduces transport, is approximated using the quenching rule $\gamma_{\text {eff }}=$ $\gamma-\omega_{e \times b}$ for the growth rate $(\gamma)$ of the corresponding modes. $^{20,21}$ The Waltz quench rule formulation ${ }^{20}$ is used inside linear solver such a way that the flow shear influences the eigenfunction $\left(\gamma=\gamma\left(\omega_{e \times b}\right)\right)$. The Waltz rule is used inside the linear solver in order to find the fastest growing mode with flowshear included. However, once the fastest growing mode is found, the Waltz quench rule is reapplied outside the linear solver. The reason that the Waltz rule is initially used inside the linear solver is that the convergence of the iterations inside the linear solver is faster for strong instabilities. The inclusion of flow shear dependence on the correlation length in Eq. (52) is the result of a generalization of an earlier derivation. ${ }^{17}$ In the absence of the flow shear in Eq. (52), the previously used correlation length formulation is recovered. It has been found that the dependence of flow shear on the correlation length ${ }^{18}$ can reproduce the experimental observation that the transport stiffness is reduced for a combination of large flow shear and small magnetic shear. $^{22}$ The reason is that for the large magnetic shear the 
radial correlation length is determined primarily by the magnetic shear, whilst for the low magnetic shear it is determined by the flow shear. The justification for using a correlation length as the inverse wavelength of the fastest growing mode is based on the fact that small eddies tear apart larger eddies so that the correlation length can normally not be much larger that the wavelength of the fastest growing mode. For shorter wavelengths, the amplitude of oscillations usually decreases rapidly thereby leaving eddies of the size of the fastest growing wavelength to dominate. ${ }^{18}$

\section{E. Transport coefficients in the Weiland model}

The generalized eigenvalue problem is established based on equations derived in the Weiland model

$$
A v=\lambda B v
$$

where $\lambda=\omega+i \gamma$ is the eigenvalue and $v$ is the corresponding eigenvector. The eigenvalues yield the real frequencies and growth rates of the modes, while the eigenvectors provide the phase and magnitude of the perturbed variables relative to one another.

Thermal and particle diffusivities in the Weiland model are calculated assuming the dominant nonlinearity is of the $\mathbf{E} \times \mathbf{B}$ convective type in the continuity or energy equation. The saturation level is obtained by balancing the linear growth rate $(\gamma)$ calculated in the Weiland model described above, with the $\mathbf{E} \times \mathbf{B}$ nonlinearity

$$
\hat{\phi} \approx \frac{1}{k_{r} \rho_{s i}} \frac{\gamma}{k_{\theta} c_{s}}=\frac{2}{R k_{r}} \frac{\gamma}{\omega_{D e}} .
$$

The ion thermal transport coefficient $\left(\chi_{i}\right)$, the electron thermal transport coefficient $\left(\chi_{e}\right)$, and the particle transport coefficient $\left(D_{n}\right)$ with electron trapping included can be calculated by employing above saturation level, the energy (continuity) equation, and Fick's law as indicated below:

$$
\begin{gathered}
\chi_{i}=\frac{1}{\eta_{i}}\left(\eta_{i}-\frac{2}{3}-\left(1-f_{t}\right) \frac{10}{9 \tau} \varepsilon_{n}-\frac{2}{3} f_{t} \Delta_{i}\right) \\
\times \frac{\gamma^{3} / k_{r}^{2}}{\left(\omega_{r}-5 / 3 \omega_{\mathrm{Di}}\right)^{2}+\gamma^{2}}, \\
\chi_{e}=f_{t} \frac{1}{\eta_{e}}\left(\eta_{e}-\frac{2}{3}-\frac{2}{3} f_{t} \Delta_{e}\right) \frac{\gamma^{3} / k_{r}^{2}}{\left(\omega_{r}-5 / 3 \omega_{\mathrm{De}}\right)^{2}+\gamma^{2}}, \\
D_{n}=f_{t} \Delta_{n} \frac{\gamma^{3} / k_{r}^{2}}{\omega_{* e}^{2}}
\end{gathered}
$$

where $\Delta_{i}, \Delta_{e}$, and $\Delta_{n}$ are all due to contributions from the trapped electrons

$$
\begin{aligned}
& \Delta_{i}= \frac{1}{N}\left\{|\hat{\omega}|^{2}\left[|\hat{\omega}|^{2}\left(\varepsilon_{n}-1\right)+\hat{\omega}_{r} \varepsilon_{n}\left(\frac{14}{3}-2 \eta_{i}-\frac{10}{3} \varepsilon_{n}\right)+\frac{5}{3} \varepsilon_{n}^{2}\left(-\frac{11}{3}+2 \eta_{e}+\frac{7}{3} \varepsilon_{n}\right)-\frac{5}{3 \tau} \varepsilon_{n}^{2}\left(1+\eta_{e}-\frac{5}{3} \varepsilon_{n}\right)\right]\right. \\
&\left.+\frac{50}{9 \tau} \hat{\omega}_{r} \varepsilon_{n}^{3}\left(1-\varepsilon_{n}\right)-\frac{25}{9 \tau} \varepsilon_{n}^{4}\left(\frac{7}{3}-\eta_{e}-\frac{5}{3} \varepsilon_{n}\right)\right\}, \\
& \Delta_{e}=\frac{1}{N}\left\{|\hat{\omega}|^{2}\left[|\hat{\omega}|^{2}\left(\varepsilon_{n}-1\right)+\hat{\omega}_{r} \varepsilon_{n}\left(\frac{14}{3}-2 \eta_{e}-\frac{10}{3} \varepsilon_{n}\right)+\frac{5}{3} \varepsilon_{n}^{2}\left(-\frac{8}{3}+3 \eta_{e}+\frac{2}{3} \varepsilon_{n}\right)\right]+\frac{50}{9 \tau} \hat{\omega}_{r} \varepsilon_{n}^{3}\left(\varepsilon_{n}-1\right)+\frac{25}{9 \tau} \varepsilon_{n}^{4}\left(\frac{7}{3}-\eta_{e}-\frac{5}{3} \varepsilon_{n}\right)\right\},(58) \\
& \Delta_{n}=\frac{1}{N}\left[|\hat{\omega}|^{2}\left(1-\varepsilon_{n}\right)-\hat{\omega}_{r} \varepsilon_{n}\left(\frac{14}{3}-2 \eta_{i}-\frac{10}{3} \varepsilon_{n}\right)-\frac{5}{3} \varepsilon_{n}^{2}\left(-\frac{11}{3}+2 \eta_{e}+\frac{7}{3} \varepsilon_{n}\right)\right],
\end{aligned}
$$

$$
N=\left(\hat{\omega}_{r}^{2}-\hat{\gamma}^{2}-\frac{10}{3} \hat{\omega}_{r} \varepsilon_{n}+\frac{5}{3} \varepsilon_{n}^{2}\right)^{2}+4 \hat{\gamma}^{2}\left(\hat{\omega}_{r}-\frac{5}{3} \varepsilon_{n}\right)^{2},
$$

where $\varepsilon_{n}=2 / g_{n}, \tau=T_{e} / T_{i}, \hat{\omega}=\omega / \omega_{* e}, \hat{\omega}_{r}=\omega_{r} / \omega_{* e}$, and $\hat{\gamma}=\gamma / \omega_{* e}$ in which $\omega_{r}$ is the real part of the frequency $\omega$ and $\gamma$ is the growth rate of the mode. Note that Eq. (55), in the absence of trapped electrons $\left(f_{t}=0\right)$, reduces to the following ion thermal diffusivity:

$$
\chi_{i}=\frac{1}{\eta_{i}}\left(\eta_{i}-\frac{2}{3}-\frac{10}{9 \tau} \varepsilon_{n}\right) \frac{\gamma^{3} / k_{r}^{2}}{\left(\omega_{r}-5 / 3 \omega_{\mathrm{Di}}\right)^{2}+\gamma^{2}} .
$$

The terms with negative signs in the Eq. (62) correspond to the ion heat pinch. However, the temperature diffusion obtained from Eq. (62) will always be outward since growth rate $\gamma$ of the mode is zero if the pinch effects dominate. It can be noted that the $\chi_{i}$ decreases in the inner region of the plasma where $\varepsilon_{n}$ is large. In the edge region of the plasma where $\varepsilon_{n} \ll 1, \eta_{i} \gg 1$, and $\gamma>\omega_{r}$, the ion thermal diffusivity in the Eq. (62) reduces to the well know mixing length expression $\gamma / k_{r}^{2}$.

The transport coefficients can be expressed analytically when impurities are included. The quasi-neutrality for perturbations is used to eliminate the main ion density perturbation in the calculation of the $\chi_{i z}$. The result is 


$$
\begin{aligned}
\chi_{i z}= & \frac{1}{\eta_{i}}\left[\eta_{i}-\frac{2}{3}-\frac{1-f_{t}}{b_{i}} \frac{10}{9 \tau} \varepsilon_{\mathrm{nh}}-\frac{2}{3} \frac{f_{t}}{b_{i}} \frac{L_{\mathrm{nh}}}{L_{\mathrm{ne}}} \Delta_{i}+\frac{2}{3} \frac{Z_{z} f_{z}}{b_{i}} \frac{L_{\mathrm{nh}}}{L_{\mathrm{nz}}} \Delta_{\mathrm{iz}}\right] \\
& \times \frac{\gamma^{3} / k_{r}^{2}}{\left(\omega_{r}-5 / 3 \omega_{\mathrm{Di}}\right)^{2}+\gamma^{2}},
\end{aligned}
$$

where $b_{i}=1-f_{z} Z_{z}$. Note that the effects that the density length scale of main ion $\left(L_{\mathrm{nh}}\right)$ and the density length scale of electron $\left(L_{\mathrm{ne}}\right)$, in general, will be different when impurities are taken into account. The impurity density response is introduced through $\Delta_{\mathrm{iz}}$, which is given by

$$
\begin{gathered}
\Delta_{\mathrm{iz}}=\frac{1}{N_{z}}\left(A_{z}+\eta_{z} B_{z}+\varepsilon_{n} C_{z}\right) \\
N_{z}=\left(\hat{\omega}_{r}^{2}-\hat{\gamma}^{2}+\frac{10}{3} \frac{\hat{\omega}_{r} \varepsilon_{n}}{Z_{z} \tau_{z}}+\frac{5}{3}\left(\frac{\varepsilon_{n}}{Z_{z} \tau_{z}}\right)^{2}\right)^{2} \\
+4 \hat{\gamma}^{2}\left(\hat{\omega}_{r}+\frac{5}{3} \frac{\varepsilon_{n}}{Z_{z} \tau_{z}}\right)^{2} \\
A_{z}=|\hat{\omega}|^{2}\left[-|\hat{\omega}|^{2}-\frac{14}{3} \frac{\varepsilon_{n}}{Z_{z} \tau_{z}} \hat{\omega}_{r}+\frac{5}{3} \frac{\varepsilon_{n}^{2}}{Z_{z} \tau_{z}}\left(\frac{1}{\tau}-\frac{11}{3 Z_{z} \tau_{z}}\right)\right] \\
+\frac{25}{9 \tau} \frac{\varepsilon_{n}^{3}}{\left(Z_{z} \tau_{z}\right)^{2}}\left(2 \hat{\omega}_{r}+\frac{7}{3} \frac{\varepsilon_{n}}{Z_{z} \tau_{z}}\right), \\
B_{z}=|\hat{\omega}|^{2}\left[2 \frac{\varepsilon_{n}}{Z_{z} \tau_{z}} \hat{\omega}{ }_{r}+\frac{5}{3} \frac{\varepsilon_{n}^{2}}{Z_{z} \tau_{z}}\left(\frac{1}{\tau}+\frac{2}{Z_{z} \tau_{z}}\right)\right]+\frac{25}{9 \tau} \frac{\varepsilon_{n}^{4}}{\left(Z_{z} \tau_{z}\right)^{3}} \\
C_{z}=|\hat{\omega}|^{2}\left[|\hat{\omega}|^{2}+\frac{10}{3} \frac{\varepsilon_{n}}{Z_{z} \tau_{z}} \hat{\omega_{r}}+\frac{5}{3} \frac{\varepsilon_{n}^{2}}{Z_{z} \tau_{z}}\left(\frac{7}{3 Z_{z} \tau_{z}}-\frac{5}{3 \tau}\right)\right] \\
+\frac{25}{9 \tau} \frac{\varepsilon_{n}^{3}}{\left(Z_{z} \tau_{z}\right)^{2}}\left(2 \hat{\omega}_{r}+\frac{7}{3} \frac{\varepsilon_{n}}{Z_{z} \tau_{z}}\right) \\
\end{gathered}
$$

where $\tau_{z}=T_{z} / Z_{z} T_{e}$. The expression for impurity diffusivity $\left(D_{z}\right)$ can be developed in an analogous manner.

Transport fluxes can be divided into diffusive and convective parts by temporarily perturbing one gradient at a time (for example, the temperature and density gradient for each species) and defining elements of the diffusion matrix as finite difference derivatives of the fluxes with respect to these gradients:

$$
(\text { Diffusion })_{i, j}=-\frac{\Delta(\text { Flux })_{i}}{\Delta(\text { gradient })_{j}} .
$$

The convective transport is then the difference between each flux and the corresponding diffusive transport

$$
(\text { Convection })_{i}=(\text { Flux })_{i}-\sum_{j}(\text { Diffusion })_{i, j}(\text { gradient })_{j} .
$$

The MMM8.1 module utilizes the same methodology as is used in trapped gyro-Landau-fluid ${ }^{39}$ and gyro-Landau-fluid ${ }^{40}$ anomalous transport models in that a set of multi-species fluid equations are linearized; eigenvalues and eigenvectors are computed; and quasi-linear estimates are computed for all the transport coefficients.

\section{DRIBM COMPONENT IN THE MMM8.1 MODEL}

The Weiland drift mode model derived in the previous section is the primary candidate for producing the turbulence that drives anomalous transport in the core of tokamak plasmas. The situation at the edge is different. Since the edge plasma is strongly influenced by collisions, it is expected that resistive mode is an important source of the edge turbulence. One of the unstable plasma modes that is believed to be responsible for edge turbulence is the RBM. ${ }^{23-35}$ Resistive ballooning modes appear to be quite unstable for typical values of the plasma edge parameters. These modes are the pressure driven modes that are localized in the regions where the magnetic field lines are concave to the plasma (along the outboard edge of tokamaks, for example). Resistive ballooning modes are unstable when the electron motion along the field lines is strongly impeded by collisions. In low-density discharges where the electron-ion collisionality is not large enough to excite resistive ballooning modes, additional physical effects, such as electron inertia, plasma impurities, induction and electron trapping, can destabilize RBMs by impeding the electron parallel dynamics.

The resistive ballooning modes can be unstable in the collisional edge region of the plasma even when the local pressure gradient is not large enough to drive ideal MHD ballooning modes. ${ }^{37}$ This is particularly true in the vicinity of the separatrix or magnetic divertor where the steep density gradients and low plasma temperature enhance the growth rate of resistive ballooning modes, in spite of the stabilizing influence of large magnetic shear near the separatrix. In contrast, the effects of increasing temperature gradient and Larmor radius are found to be stabilizing for resistive ballooning modes. ${ }^{36-38}$

The DRIBMs ${ }^{11}$ are a generalization of RBMs in which two-fluid drift effects and electron inertia are included. The DRIBM two-fluid model consists of six coupled reduced Braginskii equations: These linearized equations take into account diamagnetic effects, parallel electron and ion dynamics, electron inertia, magnetic perturbations, gyro-viscous stress terms, electron and ion equilibrium density and temperature gradients, and temperature and density perturbations.

The equations for ion continuity, total momentum (sum of electron and ion momentum equations), ion energy, vorticity, generalized Ohm's law and electron energy ${ }^{11}$ are given below using notation defined in the previous section:

$$
\begin{gathered}
\left(-\omega+\omega_{D i}\right) \hat{n}_{i}+\omega_{D i} \hat{T}_{i}+\left[\left(\omega_{D i}-\omega_{* i}\right) T_{e} / T_{i}\right. \\
\left.-k_{\theta}^{2} \rho_{s i}^{2}\left(\omega-\omega_{* i}\left(1+\eta_{i}\right)\right)\right] \hat{\phi}+k_{\|} c_{s} \hat{v}_{\| i}-i \beta \eta_{\perp} k_{\perp}^{2} \hat{p}=0 \\
\left(\omega-2 \omega_{D i}\right) \hat{v}_{\| i}-k_{\|} c_{s}\left[\left(\hat{T}_{i}+\hat{n}_{i}\right) T_{i} / T_{e}+\hat{T}_{e}+\hat{n}_{e}\right] \\
-\left[\omega_{T i}+\omega_{* i}-\left(\omega_{T e}+\omega_{* e}\right)\right] \hat{A}_{\|}=0, \\
\left(-\omega+\frac{5}{3} \omega_{D i}\right) \hat{T}_{i}+\frac{2}{3} \omega \hat{n}_{i}+\omega_{* e}\left(\eta_{i}-\frac{2}{3}\right) \hat{\phi}=0, \\
\omega_{D i}\left[\left(1+T_{e} / T_{i}\right) \hat{n}+\hat{T}_{i}+\left(T_{e} / T_{i}\right) \hat{T}_{e}\right] \\
-k_{\theta}^{2} \rho_{s i}^{2}\left[\omega-\omega_{* i}\left(1+\eta_{i}\right)\right] \hat{\phi}+\left(v_{A}^{2} / c_{s}^{2}\right) k_{\|} c_{s}\left[k_{\theta}^{2} \rho_{s i}^{2} \hat{A}_{\|}\right]=0
\end{gathered}
$$




$$
\begin{gathered}
{\left[\omega-\omega_{* e}\left(1+1.71 \eta_{e}\right)+i \eta_{\|} k_{\perp}^{2}+\omega k_{\perp}^{2} \delta_{e}^{2}\right] \hat{A}_{\|}} \\
\quad-k_{\|} c_{s}\left[\hat{\phi}-1.71 \hat{T}_{e}-\hat{n}_{e}\right]-\left(m_{e} / m_{i}\right) \omega \hat{v}_{\| i}=0 \\
\left(\omega-\frac{5}{3} \omega_{D e}+i \frac{k_{\|}^{2} v_{T e}^{2}}{0.51 \nu_{e}}\right) \hat{T}_{e}-\frac{2}{3} \omega \hat{n}_{e}-\frac{1}{2} \omega_{D e}\left(g_{T e}-\frac{2}{3} g_{n e}\right) \hat{\phi} \\
+k_{\|} c_{s}\left[\frac{0.96}{\beta_{e}} k_{\perp}^{2} \rho_{s i}^{2}-i \frac{m_{i}}{2 m_{e}} \frac{\omega_{D e}}{\nu_{e}} g_{T e}\right] \hat{A}_{\|}=0 .
\end{gathered}
$$

Here $\hat{n} \equiv \tilde{n} / n$ and $\hat{p} \equiv \tilde{p} / p$ are dimensionless forms of the density and plasma pressure perturbations, $\tilde{n}$ and $\tilde{p}$, respectively. In Eq. (75): $\delta_{e} \equiv c / \omega_{p e}$ is the electromagnetic skin depth; $c$ is the speed of light; $\omega_{p e} \equiv \sqrt{n e^{2} / \epsilon_{0} m_{e}}$ is the electron plasma frequency in which $\epsilon_{0}$ is the permittivity of free space; $k_{\|}$and $k_{\perp}$ are the parallel and perpendicular wave numbers; and $\eta_{\|}$and $\eta_{\perp}$ are longitudinal and transverse Spitizer resistivities. The following notation is used in all of these expressions:

$$
\begin{aligned}
\omega_{T_{e}} & \equiv k_{\theta} \frac{T_{e} g_{T e}}{e B R}, & \omega_{T_{i}} & \equiv-k_{\theta} \frac{T_{i} g_{T i}}{e B R} \\
g_{T j} & \equiv-R \frac{\hat{x} \cdot \nabla T_{j}}{T_{j}}, & v_{t j} & \equiv \sqrt{\frac{T_{j}}{m_{j}}},
\end{aligned}
$$

where $\omega_{T j}, g_{T j}$, and $v_{t j}$ are the temperature gradient drift frequency, the normalized temperature gradient, and thermal velocity for species $j$, respectively.

The DRIBM is developed for computing the electron and ion thermal and particle diffusivities in the axisymmetric tokamak edge plasmas. As noted above, the DRIBM model is based on the linearized multifluid Braginskii equations for the time evolving perturbed density, velocity, and temperature of thermal ions and electrons. The derivation also uses Ampere's and Faraday's law for the perturbed electric and magnetic fields. The electron trapping, the displacement current, and the effects of impurity ions are neglected. A mixing length approximation, described below, is used to compute the thermal and particle fluxes from the eigenvalue and eigenvector solutions. The thermal and particle fluxes driven by DRIBMs have different magnitudes due to the fact that the temperature and density perturbations have different amplitudes and phases relative to the perturbed convective velocity.

\section{A. Thermal and particle transport fluxes in the DRIBM model}

The DRIBM equations (71)-(76) are used to establish the generalized eigenvalue problem (53). In the short poloidal wavelength (high poloidal mode number) limit, these equations become localized to each flux surface. Computational techniques are used to find the eigenvalues and eigenfunctions of these linearized equations. The anomalous particle and thermal transport fluxes can be computed directly from the eigenvalues and the eigenvectors. The particle flux, produced by the perturbed $\mathbf{E} \times \mathbf{B}$ motion of the plasma, is given by

$$
\Gamma_{n}=\tilde{n} \tilde{v}_{E}^{*}+\text { c.c. }=2(\operatorname{Re}[\tilde{n}] \operatorname{Im}[\tilde{\phi}]-\operatorname{Im}[\tilde{n}] \operatorname{Re}[\tilde{\phi}]) k_{\theta} / B,
$$

where $\tilde{v}_{E}^{*}$ represents complex conjugate of the perturbed $\mathbf{E} \times \mathbf{B}$ drift. Note, even though magnetic perturbations are included in the models used to compute the eigenvalues and eigenvectors, the fluxes include only the electrostatic $\mathbf{E} \times \mathbf{B}$ drift computed from these eigenvalues and eigenvectors. The saturation level of turbulence is computed by balancing the linear growth rate with the nonlinearity (54) which results in a particle flux given by

$$
\frac{\Gamma_{n}}{n} \frac{R k_{r}^{2}}{\omega_{D e}}=4 \hat{\gamma}^{2}(\operatorname{Re}[\tilde{n}] \operatorname{Im}[\tilde{\phi}]-\operatorname{Im}[\tilde{n}] \operatorname{Re}[\tilde{\phi}]) /|\hat{\phi}|^{2},
$$

where $\hat{\gamma}=\gamma / \omega_{D e}$ is the growth rate normalized by the electron magnetic drift frequency. Similarly the ion heat flux is given by

$$
\frac{\Gamma_{i}}{n T_{i}} \frac{R k_{r}^{2}}{\omega_{D e}}=4 \hat{\gamma}^{2}\left(\operatorname{Re}\left[\hat{n}+\hat{T}_{i}\right] \operatorname{Im}[\hat{\phi}]-\operatorname{Im}\left(\hat{n}+\hat{T}_{i}\right) \operatorname{Re}[\hat{\phi}]\right) /|\hat{\phi}|^{2} .
$$

Equation (80) is used together with Fick's law, $\Gamma_{i}=-\chi_{i}\left(d T_{i} / d x\right)$, to compute an effective ion thermal diffusivity $\chi_{i}$. The turbulence is assumed to be isotropic in the sense that the poloidal and radial wavenumbers are the same. It is found that the fastest growing mode for DRIBM model occurs in the vicinity $k_{r} \rho_{s}=0.2$. Therefore, $k_{r} \rho_{s}=0.2$ has been used in the DRIBM component of an earlier version of the Multi-Mode model. The procedure for separating diffusion and convection shown in Eq. (70) can also be used for the DRIBM model.

\section{ETG COMPONENT IN THE MMM8.1 MODEL}

Transport driven by short wavelength ETG modes is another component within the MMM8.1 transport model. Since ETG modes have very short wavelength-comparable to the electron gyro-radius rather than the ion gyro-radiusETG modes drive essentially only electron thermal transport, with almost no contribution to ion thermal, particle, or momentum transports. The model chosen for the ETG component $^{45,46}$ is based on a quasi-linear model by Horton ${ }^{9}$ modified by an ETG mode threshold computed by Jenko. ${ }^{15}$ The Horton ETG model was developed as a generalization of a hydrodynamic theory for short wavelength ETG turbulence with electromagnetic effects included. The model was then calibrated using data from fast wave electron heated Tore Supra discharges with hot electrons. ${ }^{10}$

In this model for ETG turbulence, it is assumed that the ETG mode is a toroidal version of the lower hybrid drift mode driven unstable by charge separation due to the unfavorable $\nabla B$ and curvature electron drift in the presence of an electron temperature gradient. The resulting instability can drive short wavelength drift wave turbulence resulting in electron thermal transport in tokamaks. In the non-linear regime, it is assumed that the turbulence is driven in two space scale regimes.

The short wavelength regime is electrostatic, where the maximum linear growth pumps energy into the electrostatic turbulence, while the long wavelength regime is neutrally stable. The ETG fluctuation spectrum mode couples energy to longer space scale fluctuations. This inverse cascade, or 
mode coupling of shorter space scale electrostatic fluctuations to longer space scales, drives a coupling to the parallel vector potential $A_{\|}$fluctuations at the scale of electromagnetic skin depth $c / \omega_{\text {pe }}$. At this scale, the fluctuations become neutrally stable electromagnetic vortices. This secondary driven electromagnetic turbulence is expected to lead to a large stochastic diffusion of the trapped electrons. Stochastic transport studies indicate that, once the turbulence level reaches the mixing length level, the electron diffusivity is insensitive to the details of the fluctuation spectrum and, under these conditions, the electron diffusivity is governed by a random walk of the trapped electrons over the skin depth correlation length, $c / \omega_{\text {pe }}$, at the rate of the decorrelation frequency, which is of the order of bounce frequency of the trapped electrons. For the long wavelength part of the spectrum, stochasticity occurs due to the overlap of the vortex circulation frequency with the parallel bounce frequency, which produces a strong diffusion of the trapped electrons and a weak diffusion of the passing electrons.

The Horton model includes an electrostatic contribution, which is analogous to the ITG electrostatic model, and an electromagnetic contribution, which in the Horton model describes non-isotropic mode structure when the turbulence mixing length, $l_{c, e}=q \rho_{e} R / L_{T_{e}}$ is greater than the electron skin depth $\delta_{e}=c / \omega_{p e}$. In this model, the critical threshold gradient for the transport driven by ETG modes, based upon linear toroidal gyrokinetic simulations, is given by the formula

$$
\begin{aligned}
\left(\frac{R}{L_{T_{e}}}\right)_{\text {crit }}= & \max \left[\left(1+\frac{Z_{\mathrm{eff}} T_{e}}{T_{i}}\right)\left(1.33+\frac{1.91 s}{q}\right)(1-1.5 \epsilon)\right. \\
& \left.\times\left(1+0.3 \epsilon \frac{d \kappa}{d \epsilon}\right), 0.8 \frac{R}{L_{n}}\right] .
\end{aligned}
$$

where $\epsilon=r_{0} / R_{0}$ is the inverse aspect ratio.

The effective ETG electron thermal diffusivity in the electrostatic limit $\left(l_{c, e}<\delta_{e}\right)$, including the Jenko threshold, is given by

$$
\chi_{e}^{\mathrm{es}}=0.06 q^{2}\left(\frac{R}{L_{T_{e}}}\right)^{3 / 2} \frac{\rho_{e}^{2} v_{t h, e}}{R} \max \left[\left[\frac{R}{L_{T_{e}}}-\left(\frac{R}{L_{T_{e}}}\right)_{\mathrm{crit}}\right], 0\right],
$$

where $v_{t h, e}$ is the thermal velocity of the electrons and $\rho_{e}$ is the electron gyroradius. In the expression for $\chi_{e}^{e s}$, the critical gradient given in Ref. 10 is replaced by Eq. (81). The effective ETG electron thermal diffusivity in the electromagnetic limit $\left(l_{c, e}>\delta_{e}\right)$, which is also modified in order to include the Jenko threshold, is given by

$$
\chi_{e}^{\mathrm{em}}=0.06 \delta_{e}^{2} v_{t h, e} \frac{\sqrt{R / L_{T_{e}}}}{R} \max \left[\tanh \left[\frac{R}{L_{T_{e}}}-\left(\frac{R}{L_{T_{e}}}\right)_{\mathrm{crit}}\right], 0\right] .
$$

The electromagnetic part of the ETG diffusivity is zero below the threshold or for negative temperature gradient. It was found that electromagnetic limit of the ETG mode provides an important contribution, particularly in the region near the magnetic axis. ${ }^{46}$

\section{TOROIDAL MOMENTUM TRANSPORT}

It is important to predict the plasma toroidal rotation frequency profile in tokamaks because the rotation effects can have a significant impact on plasma confinement, fusion power production, and instabilities such as resistive wall modes and neoclassical tearing modes. The toroidal rotation frequency, which is a measure of the net plasma velocity, can affect thermal confinement through the flow shear stabilization of turbulent transport. In the core of H-mode neutral beam heated discharges, the gradient of the toroidal rotation frequency produces the largest contribution to the flow shear rate.

The toroidal momentum transport can be calculated using the parallel electromagnetic ion momentum Eq. (18) with the ion stress tensor and zero order background flow included

$$
\begin{aligned}
\delta v_{\| i}= & \frac{-k_{\theta} D_{B}}{\omega-2 \omega_{D i}} \frac{d V_{\| 0}}{d r} \hat{\phi}+\frac{k_{\|} c_{s}^{2}+\left(T_{e} / T_{i}\right) V_{\| 0} \omega_{D i}}{\omega-2 \omega_{D i}} \\
& \times\left[\hat{\phi}+\frac{T_{i}}{T_{e}} \frac{\delta p_{i}}{p_{i}}-\frac{\omega-\omega_{* i}\left(1+\eta_{i}\right)}{c k_{\|}} \hat{A}_{\|}\right],
\end{aligned}
$$

where $D_{B} \equiv \rho_{s} c_{s}$. The toroidal momentum flux, $\Gamma_{\phi}$, is calculated using the following expression:

$$
\Gamma_{\phi}=m_{i} n_{i} \operatorname{Re}\left[\left\langle v_{E}^{*} v_{\phi}\right\rangle\right]=m_{i} n_{i} \chi_{\phi} \nabla V_{\phi},
$$

where $*$ denotes complex conjugate, $\chi_{\phi}$ is the momentum diffusivity, and $v_{\phi}$ is the toroidal velocity. The toroidal velocity is approximated as $v_{\phi} \approx v_{\|}-\left(B_{\theta} / B_{\phi}\right) v_{\theta}$, in which $v_{\theta}$ is the poloidal component of the convected velocity, $B_{\phi}$ and $B_{\theta}$ are the toroidal and poloidal components of the magnetic field, and $V_{\phi}$ is the toroidal flow velocity.

\section{A. Diagonal toroidal and poloidal momentum transport}

The first term on the right hand side of Eq. (84) is the $\mathbf{E} \times \mathbf{B}$ convection of the background velocity that gives the diagonal momentum flux. The second term will provide the off-diagonal part of the parallel momentum equation. The diagonal momentum flux is calculated using

$$
\Gamma_{\phi D}=m_{i} n_{i} \operatorname{Re}\left[\left\langle v_{E r}^{*} v_{\phi, D}\right\rangle\right], \quad \text { where } v_{E r}^{*}=i k_{\theta} D_{B} \hat{\phi}^{*}
$$

The velocity $v_{\phi, D}$, ignoring the poloidal flow in the first term of the right hand side of Eq. (84), can be written as

$$
v_{\phi, D}=\frac{-k_{\theta} D_{B}}{\omega-2 \omega_{D i}} \frac{d V_{\phi}}{d r} \hat{\phi} .
$$

The following result for the diagonal toroidal momentum diffusivity can be obtained by employing Ficks' law $\left(\chi_{\phi, D}=-\Gamma_{\phi, D} / d_{r} V_{\phi}\right)$ and by assuming that the $\mathbf{E} \times \mathbf{B}$ convection is the main nonlinear saturation mechanism and that the normalized potential fluctuation $\hat{\phi}$ is estimated by Eq. (54)

$$
\chi_{\phi, D}=\frac{\gamma^{3} / k_{r}^{2}}{\left(\omega_{r}-2 \omega_{D i}\right)^{2}+\gamma^{2}}
$$

where $\omega_{r}$ is the real part of the frequency $\omega$. The above diagonal toroidal momentum diffusivity expression can also be 
used for the diagonal poloidal momentum diffusivity. A result similar to Eq. (88) was obtained previously by kinetic orbit integration. $^{41}$

The diagonal component of the ion thermal diffusivity can be obtained employing a similar approach

$$
\chi_{i}=\frac{\gamma^{3} / k_{r}^{2}}{\left(\omega_{r}-5 / 3 \omega_{D i}\right)^{2}+\gamma^{2}} .
$$

Note that the diagonal part of the momentum diffusivity in Eq. (88) is similar to the diagonal part of the ion thermal diffusivity in Eq. (89). The expressions for both $\chi_{\phi}$ and $\chi_{i}$ have resonance terms in the denominator due to toroidicity but slightly different coefficients. It can be seen that the anomalous transport is reduced due to the presence of a real frequency (non-Markovian effect). However, for $\gamma \gg \omega_{r}$, the Kadomstev mixing length transport $\left(\gamma / k_{r}^{2}\right)$ can be recovered. It has been found that the Kadomstev mixing length estimate is good for typical experimental situations as long as there is only one dominant instability. However, in a case where several instabilities are present, non-Markovian effects in Eqs. (88) and (89), in combination with the toroidal Doppler shift, are required in order to separate out how different types of instabilities affect different transport coefficients. The Doppler shift $(5 / 3) \omega_{D i}$, in the expression for the ion thermal diffusivity, is due to the presence of diamagnetic heat flow term in the ion energy equation. The physical reason for the Doppler shift is that ions move with the magnetic drift velocity. Since the resonance $\omega-2 \omega_{D i}$ in the $\chi_{\phi}$ expression is close to the resonance $\omega-(5 / 3) \omega_{D i}$ in the diagonal part of $\chi_{i}$, the ratio $\chi_{\phi} / \chi_{i}$ corresponding to the diagonal transport far from the marginal stability is close to unity. Close to the marginal stability, as for example near the axis in tokamak where the real frequency approaches to $(5 / 3) \omega_{D i}$, the ratio $\chi_{\phi} / \chi_{i}$ is greater than unity.

\section{B. Off-diagonal toroidal momentum transport}

The off-diagonal toroidal momentum flux, $\Gamma_{\phi, O D}$, has a parallel and perpendicular components

$$
\Gamma_{\phi, O D}=\Gamma_{\phi, O D \|}+\Gamma_{\phi, O D \perp} .
$$

The parallel component, $\Gamma_{\phi, O D \|}$, can be calculated by utilizing the second term in the right hand side of Eq. (84), which is the off-diagonal contribution from the Reynolds stress, parallel velocity

$$
\begin{aligned}
\Gamma_{\phi, O D \|}= & m_{i} n_{i} \operatorname{Re}\left\langle v_{E r}^{*} \frac{k_{\|} c_{s}^{2}+\left(T_{e} / T_{i}\right) V_{\phi} \omega_{D i}}{\omega-2 \omega_{D i}}\right. \\
& \left.\times\left[\hat{\phi}+\frac{T_{i}}{T_{e}} \frac{\delta p_{i}}{p_{i}}-\frac{\omega-\omega_{* i}\left(1+\eta_{i}\right)}{c k_{\|}} \hat{A}_{\|}\right]\right\rangle .
\end{aligned}
$$

The off diagonal parallel part of toroidal momentum diffusivity $\left(\chi_{\phi, O D \|}\right)$ is calculated by utilizing Ficks' law

$$
\begin{aligned}
\chi_{\phi, O D \|} \approx & -\frac{k_{\theta} D_{B}}{d V_{\phi} / d r}\left(\left\langle k_{\|}\right\rangle c_{s}^{2}-V_{\phi} \omega_{D e}\right) \\
& \times \operatorname{Re}\left\langle\frac{i \hat{\phi}^{*}}{\omega-2 \omega_{D i}}\left[\hat{\phi}+\frac{T_{i}}{T_{e}} \frac{\delta p_{i}}{p_{i}}-\frac{\omega-\omega_{* i}\left(1+\eta_{i}\right)}{c k_{\|}} \hat{A}_{\|}\right]\right\rangle,
\end{aligned}
$$

where $\left\langle k_{\|}\right\rangle$in Eq. (92) is an average parallel wave number over the mode profile and can be written as ${ }^{42,43}$

$$
\begin{aligned}
\left\langle k_{\|}\right\rangle= & -\frac{1}{q R} \\
& \times \frac{0.5(\hat{\omega}+5 / 3) \kappa_{1}+h\left(\hat{\omega}-\hat{\omega}_{r k}\right) \kappa_{2}}{\hat{\omega}(1+5 / 3)+\left(1 / \tau \varepsilon_{n}\right)\left(\eta_{i}-2 / 3\right)+(5 / 3 \tau)(1+1 / \tau)},
\end{aligned}
$$

where

$$
\begin{aligned}
\kappa_{1} & =\left(\frac{s k_{\theta}^{2} R q}{\omega_{c i}} \frac{d V_{\| 0}}{d y}+2 \tau^{2} q k_{\theta} \rho_{s} \frac{V_{\| 0}}{c_{s}}\right) \frac{\omega}{\omega-2 \omega_{D i}} ; \\
\kappa_{2} & =\frac{k_{\theta} \frac{d V_{\theta}}{d y}+k_{\|} \frac{d V_{\| 0}}{d y}}{\omega_{* e}}
\end{aligned}
$$

and

$$
\tau=\frac{T_{e}}{T_{i}}, \quad h=4 k_{\theta}^{2} \rho_{s}^{2} \frac{\omega}{\omega_{* e}}\left(\frac{q}{\varepsilon_{n}}\right)^{2} .
$$

In the definitions for $\kappa_{1}$ and $\kappa_{2}$ in Eq. (94), note the contributions of toroidal and poloidal flow velocities gradients, $\hat{\omega}_{r k}$ is the normalized real part of the linear eigenvalue in the absence of rotation. Thus, $\left\langle k_{\|}\right\rangle$is generated by poloidal as well as toroidal flow shear together with curvature effects from the stress tensor. Based on the assumption that $a / q R \propto k_{\theta} \rho_{s}$, an average $\left\langle k_{\|}\right\rangle$can be estimated as

$$
\left\langle k_{\|}\right\rangle \propto \frac{a}{L} \frac{1}{q R},
$$

where $L$ is the length scale of toroidal and poloidal flows.

The other off-diagonal component of the toroidal momentum flux $\Gamma_{\phi, O D \perp}$ in Eq. (90) and associated effective diffusivity $\chi_{\phi, O D \perp}$ can be calculated using the toroidal component of the $\mathbf{E} \times \mathbf{B}$ drift velocity

$$
v_{E, \phi}=-i(\epsilon / q) k_{r} D_{B} \hat{\phi},
$$

and the toroidal component of the diamagnetic drift velocity

$$
v_{* i, \phi}=-i\left(T_{i} / T_{e}\right)(\epsilon / q) k_{r} D_{B} \hat{P}_{i},
$$

where $\epsilon / q=B_{\theta} / B_{\phi}$. The perpendicular contribution from the Reynolds stress

$$
\chi_{\phi, O D \perp}=-\frac{\epsilon}{q} \frac{k_{r} k_{\theta} D_{B}^{2}}{d V_{\phi} / d r} \operatorname{Re}\left\langle\hat{\phi}^{*}\left(\hat{\phi}+\frac{1}{\tau} \hat{P}_{i}\right)\right\rangle .
$$

The total toroidal effective momentum diffusivity $\chi_{\phi}=$ $\chi_{\phi, D}+\chi_{\phi, O D \|}+\chi_{\phi, O D \perp}$ can be computed by adding Eqs. (88), (92), and (98) as indicated in Ref. 44

$$
\begin{aligned}
\chi_{\phi}= & \frac{\gamma^{3} / k_{r}^{2}}{\left(\omega_{r}-2 \omega_{D i}\right)^{2}+\gamma^{2}}-\frac{\epsilon}{q} \frac{k_{r} k_{\theta} D_{B}^{2}}{d V_{\phi} / d r} \operatorname{Re}\left\langle\hat{\phi}^{*}\left(\hat{\phi}+\frac{1}{\tau} \hat{P}_{i}\right)\right\rangle \\
& -\frac{k_{\theta} D_{B}}{d V_{\phi} / d r}\left(\left\langle k_{\|}\right\rangle c_{s}^{2}-V_{\phi} \omega_{D e}\right) \\
& \times \operatorname{Re}\left\langle\frac{i \hat{\phi}^{*}}{\omega-2 \omega_{D i}}\left[\hat{\phi}+\frac{T_{i}}{T_{e}} \frac{\delta p_{i}}{p_{i}}-\frac{\omega-\omega_{* i}\left(1+\eta_{i}\right)}{c k_{\|}} \hat{A}_{\|}\right]\right\rangle .
\end{aligned}
$$




\section{Off-diagonal poloidal momentum transport}

The off-diagonal poloidal momentum transport can be calculated using the following expression:

$$
\Gamma_{\theta}=\operatorname{Re}\left\langle v_{E r}^{*} v_{\theta}\right\rangle=-\chi_{\theta, O D \perp} \frac{\partial V_{\theta}}{\partial r},
$$

where $\chi_{\theta, O D \perp}$ is the off-diagonal poloidal momentum diffusivity and $V_{\theta}$ is the poloidal flow velocity, and

$$
v_{\theta}=v_{E \theta}+v_{* i \theta},
$$

in which

$$
v_{E \theta}=i k_{r} D_{B} \hat{\phi}
$$

is the poloidal component of the $\mathbf{E} \times \mathbf{B}$ drift velocity and

$$
v_{* i \theta}=-i\left(T_{i} / T_{e}\right) k_{r} D_{B} \hat{P}_{i}
$$

is the poloidal component of the diamagnetic drift velocity. It follows from Eq. (100) that the off diagonal poloidal momentum diffusivity can be written as

$$
\chi_{\theta, O D \perp}=-\frac{k_{r} k_{\theta} D_{B}^{2}}{d V_{\theta} / d r} \operatorname{Re}\left\langle\hat{\phi}^{*}\left(\hat{\phi}+\frac{1}{\tau} \hat{P}_{i}\right)\right\rangle .
$$

The momentum transport and flow-shear suppression features of the new MMM8.1 model will be utilized to compute the toroidal and poloidal angular frequency profiles that are important in the computation of the internal and edge transport barriers. Note that in the earlier version of Multi-Mode model, the electromagnetic effects in the toroidal momentum diffusivity were not included. It has been seen in a numerical study that electromagnetic effects on toroidal momentum transport can increase the toroidal momentum pinch and are sometimes strong enough to make the toroidal momentum flux inward. ${ }^{44}$ It is important to understand the poloidal rotation in the ITER plasmas, in which the toroidal rotation is expected to be low. In those discharges poloidal rotation and pressure gradient will primarily contribute to the radial electric field. The gradient of the radial electric field will provide flow shear, which can provide suppression of mode turbulence and can result in an internal or edge transport barrier.

\section{SUMMARY}

A theory based Multi-Mode anomalous transport module version 8.1 (MMM8.1) is derived, which can be used to predict ion thermal, electron thermal, particle and momentum transports. The MMM8.1 transport module consists of a combination of components corresponding to different modes of transport driven by turbulence and collisional effects. The MMM8.1 module includes a model for ion temperature gradient, trapped electron, kinetic ballooning, peeling, collisionless and collision dominated magnetohydrodynamics modes as well as a model for electron temperature gradient modes and a model for drift resistive inertial ballooning modes. The effects of impurities, trapping, collisionality, magnetic shear, flow shear, finite beta, elongated plasma shaping, and $T_{i} \neq T_{e}$ fast ions (which are assumed to not take part in the perturbation) are incorporated in the MMM8.1 module. Magnetic drifts are used in the closure of the Braginskii equations. These equations are assumed to be localized on each flux surface. Turbulence in the model is assumed to be isotropic. Diagonal and non-diagonal components of toroidal and poloidal momentum transport are derived. Dependence of drift wave turbulence correlation length on magnetic $q$, shear, elongation, temperature ratio, and flow shear has been introduced into the Weiland drift wave transport model. Inclusion of flow shear dependence in correlation length reproduces the experimental observation that stiffness is reduced for a combination of large flow shear and small magnetic shear. In contrast with the MMM7.1 transport module, the MMM8.1 transport module includes a number of new features: Peeling modes, poloidal momentum diffusivity, electromagnetic effects on toroidal momentum diffusivity, and dependence of turbulence correlation length on flow shear.

\section{ACKNOWLEDGMENTS}

This paper is dedicated to the memory of Glenn Bateman whose perseverance and tireless efforts were instrumental in establishing the merits of Multi-Mode transport model for describing the evolution of magnetically confined fusion plasmas.

${ }^{1}$ A. H. Kritz, G. Bateman, J. Kinsey, A. Pankin, T. Onjun, A. Redd, D. McCuneb, C. Ludescherb, A. Pletzerb, R. Andreb, L. Zakharovb, L. Lodestroc, L. D. Pearlsteinc, R. Jongc, W. Houlbergd, P. Strandd, J. Wileye, P. Valanjue, H. St. John, R. Waltz, J. Mandrekasg, T. K. Mauh, J. Carlssoni, and B. Braamsj, Comput. Phys. Commun. 164, 108 (2004).

${ }^{2}$ J. R. Cary, J. Candy, R. H. Cohen, S. Krasheninnikov, D. C. McCune, D. J. Estep, J. Larson, A. D. Malony, A. Pankin, P. H. Worley J. A. Carlsson, A. H. Hakim, P. Hamill, S. Kruger, M. Miah, S. Muzsala, A. Pletzer, S. Shasharina, D. Wade-Stein, N. Wang, S. Balay, L. McInnes, H. Zhang, T. Casper, L. Diachin, T. Epperly, T. D. Rognlien, M. R. Fahey, J. Cobb, A. Morris, S. Shende, G. W. Hammett, K. Indireshkumar, D. Stotler, and A. Y. Pigarov, J. Phys.: Conf. Ser. 125, 012040 (2008).

${ }^{3}$ G. V. Pereverzev and P. N. Yushmanov, ASTRA; Automated system for transport analysis, IPP Report 5/98, 2002.

${ }^{4}$ S. C. Jardin, J. L. DeLucia, and N. J. Pomphrey, J. Comput. Phys. 66, 481 (1986).

${ }^{5}$ D. B. Batchelor, E. DÁzevedo, G. Bateman, D. E. Bernholdt, L. A. Berry, P. T. Bonoli, R. Bramley, J. Breslau, M. Chance, J. Chen, M. Choi, W. Elwasif, G.-Y. Fu, R. Harvey, W. A. Houlberg, E. Jaeger, S. C. Jardin, D. Keyes, S. Klasky, S. Kruger, L. Ku, D. McCune, J. Ramos, D. P. Schissel, D. Schnack, and J. Wright, "Integrated physics advances in simulation of wave interactions with extended mhd phenomena," J. Phys.: Conf. Ser. 78, 012003 (2007).

${ }^{6}$ T. Rafiq, A. H. Kritz, G. Bateman, C. Kessel, D. C. McCune, and R. V. Budny, Phys. Plasmas 18, 112508 (2011).

${ }^{7}$ A. H. Kritz, T. Rafiq, C. Kessel, G. Bateman, D. C. McCune, R. V. Budny, and A. Y. Pankin, Nucl. Fusion 51, 123009 (2011).

${ }^{8} \mathrm{~J}$. Weiland, Stability and Transport in Magnetic Confinement System Springer Series on Atomic, Optical and Plasma Physics Vol. 71 (Springer, 2012).

${ }^{9}$ W. Horton, B.-G. Hong, and W. M. Tang, Phys. Fluids 31, 2971 (1988).

${ }^{10}$ W. Horton, P. Zhu, T. Hoang, T. Aniel, M. Ottaviani, and X. Garbet, Phys. Plasmas 7, 1494 (2000).

${ }^{11}$ T. Rafiq, G. Bateman, A. H. Kritz, and A. Y. Pankin, Phys. Plasmas 17, 082511 (2010).

${ }^{12}$ J. Weiland, K. Crombe, P. Mantica, V. Naulin, T. Tala, and JET-EFDA Contributors, IFP-CNR-Chalmers Workshop on Nonlinear Phenomena in Fusion Plasmas, AIP Conf. Proc. 1392, 85 (2011).

${ }^{13}$ F. D. Halpern, A. Eriksson, G. Bateman, A. H. Kritz, A. Pankin, C. M. Wolfe, and J. Weiland, Phys. Plasmas 15, 012304 (2008). 
${ }^{14}$ G. Bateman, A. H. Kritz, J. E. Kinsey, A. J. Redd, and J. Weiland, Phys. Plasmas 5, 1793 (1998).

${ }^{15}$ F. Jenko, W. Dorland, and G. W. Hammett, Phys. Plasmas 8, 4096 (2001).

${ }^{16}$ J. Nilsson and J. Weiland, Nucl. Fusion 34, 803 (1994)

${ }^{17} \mathrm{~J}$. Weiland and I. Holod, Phys. Plasmas 12, 012505 (2005).

${ }^{18} \mathrm{~J}$. Weiland and P. Mantica, JET-EFDA Contributors, in 38th EPS Conference on Plasma Physics (2011), P5.130.

${ }^{19}$ J. E. Kinsey, R. E. Waltz, and J. Candy, Phys. Plasmas 14, 102306 (2007).

${ }^{20}$ R. E. Waltz, G. D. Kerbel, and J. Milovich, Phys. Plasmas 1, 2229 (1994).

${ }^{21}$ G. M. Staebler, R. E. Waltz, and J. C. Wiley, Nucl. Fusion 37, 287 (1997).

${ }^{22}$ P. Mantica D. Strintzi, T. Tala, C. Giroud, T. Johnson, H. Leggate, E. Lerche, T. Loarer, A. G. Peeters, A. Salmi, A. S. Sharapov, D. Van Eester, P. C. de Vries, L. Zabeo, and K.-D. Zastrow, Phys. Rev. Lett. 102, 175002 (2009).

${ }^{23}$ A. H. Glasser, J. M. Greene, and J. L. Johnson, Phys. Fluids 18, 875 (1975).

${ }^{24}$ G. Bateman and D. Nelson, Phys. Rev. Lett. 41, 1804 (1978).

${ }^{25}$ D. Correa-Restrepo, Z. Naturforsch. A 37, 848 (1982).

${ }^{26}$ W. Anthony Cooper and M. Cristina Depassier, Physical Rev. A 32, 3124 (1985).

${ }^{27}$ P. H. Diamond, P. L. Similon, T. C. Hender, and B. A. Carreras, Phys. Fluids 28, 1116 (1985).

${ }^{28}$ J. W. Connor and R. J. Hastie, Plasmas Phys. Controlled Fusion 27, 621 (1985).

${ }^{29}$ X. Llobet, H. L. Berk, and M. N. Rosenbluth, Phys. Fluids 30, 2750 (1987).

${ }^{30}$ A. Zeiler, J. F. Drake, and B. Rogers, Phys. Plasmas 4, 2134 (1997).

${ }^{31}$ P. N. Guzdar, J. F. Drake, D. McCarthy, A. B. Hassam, and C. S. Liu, Phys. Fluids B 5, 3712 (1993).

${ }^{32}$ B. N. Rogers and J. F. Drake, Phys. Plasmas 6, 2797 (1999).
${ }^{33}$ J. R. Myra, D. A. D'Ippolito, X. Q. Xu, and R. H. Cohen, Phys. Plasmas 7, 4622 (2000).

${ }^{34}$ R. J. Hastie, J. J. Ramos, and F. Porcelli, Phys. Plasmas 10, 4405 (2003).

${ }^{35}$ T. Rafiq, C. C. Hegna, J. D. Callen, and A. H. Kritz, Phys. Plasmas 16, 102505 (2009).

${ }^{36}$ T. Rafiq, C. C. Hegna, J. D. Callen, and A. H. Kritz, Phys. Plasmas 17, 022502 (2010).

${ }^{37}$ A. E. Hubbard, R. L. Boivin, R. S. Granetz, M. Greenwald, J. W. Hughes, I. H. Hutchinson, J. Irby, B. LaBombard, Y. Lin, E. S. Marmar, A. Mazurenko, D. Mossessian, E. Nelson-Melby, M. Porkolab, J. A. Snipes, J. Terry, S. Wolfe, S. Wukitch, B. A. Carreras, and V. Klein, Phys. Plasmas 8, 2033 (2001).

${ }^{38}$ R. Singh, H. Nordman, J. Anderson, and J. Weiland, Phys. Plasmas 5, 3669 (1998).

${ }^{39}$ G. M. Staebler, J. E. Kinsey, and R. E. Waltz, Phys. Plasmas 14, 055909 (2007).

${ }^{40}$ R. E. Waltz, G. M. Staebler, W. Dorland, G. W. Hammett, M. Kotschenreuther, and J. A. Koning, Phys. Plasmas 4, 2482 (1997).

${ }^{41}$ A. Zagorodny and J. Weiland, Phys. Plasmas 6, 2359 (1999).

${ }^{42}$ A. Davydova and J. Weiland, Phys. Plasmas 7, 243 (2000).

${ }^{43}$ J. Weiland, R. Singh, H. Nordman, P. Kaw, A. G. Peeters, and D. Strinzi, Nucl. Fusion 49, 065033 (2009).

${ }^{44}$ M. A. Mahmood, A. Eriksson, and J. Weiland, Phys. Plasmas 17, 122310 (2010).

${ }^{45}$ G. Bateman, A. H. Kritz, A. Y. Pankin, T. Onjun, J. E. McElhenny, and C. MacDonald, Plasma Phys. Controlled Fusion 48, A93 (2006).

${ }^{46}$ T. Rafiq, A. Y. Pankin, G. Bateman, A. H. Kritz, and F. D. Halpern, Phys. Plasmas 16, 032505 (2009). 